\title{
Klippel-Trenaunay Syndrome: A Case Report and Literature Review
}

\author{
Wei Zhi MAK, Qiantai HONG, Sadhana CHANDRASEKAR, Glenn Wei Leong TAN, Zhiwen \\ Joseph LO* \\ Vascular Surgery Service, Department of General Surgery, Tan Tock Seng Hospital Singapore
}

*Corresponding Author: Zhiwen Joseph LO, Vascular Surgery Service, Department of General Surgery, Tan Tock Seng Hospital, 11 Jalan Tan Tock Seng, Singapore, E-mail: zhiwen@ gmail.com

\begin{abstract}
Klippel Trenaunay Syndrome (KTS) is a rare, sporadic vascular malformation chracterised by its clinical triad of: port wine stains, varicosities and limb hypertrophy. Here, we present a case of a 51-year-old woman who presented with these symptoms. We will discuss the hypothesized etiology and recommended management of KTS with a literature review.
\end{abstract}

Keywords: Klippel-Trenaunay Syndrome

\section{INTRODUCTION}

The eponymously named Klippel-Trenaunay Syndrome (KTS) is a rare cutaneous vascular condition that was first described in 1900 by two French physicians Klippel and Trenaunay, who first noticed this syndrome in two patients who presented with a port wine stain and varicosities of an extremity associated with hypertrophy of soft and bony tissue. They termed the syndrome "naevus vasculosus osteohypertrophicus". In 1907, Frederick Parkes Weber, unaware of Klippel and Trenaunay's report, described a patient with the three aforementioned symptoms as well as an arteriovenous malformation of the affected extremity. $\mathrm{He}$ termed the process hemangiectatic hypertrophy and more commonly known today as Parkes Weber Syndrome (PWS). KTS must be distinguished from PWS which has a hallmark feature of AVFs [1].

\section{CASE REPORT}

A 51 year-old Caucasian lady presented to the Vascular Surgery clinic for left lower limb varicosities. She complains of extensive skin stains since birth, with worsening left lower limb swelling and varicosities for the past 20 years. She denies any prior superficial thrombophlebitis, deep vein thrombosis, pulmonary embolism, venous ulcerations or rectal bleeding. As she is a home-maker, the limb swelling and varicosities do not affect her daily activities. Upon examination, the patient has extensive port wine stains over her left arm, torso and left lower limb which were associated with left lower limb varicosities and left thigh hypertrophy (Figures 1 to 4 ). The patient has the Klippel-Trenaunay Syndrome clinical trial of port wine stains, varicosities and limb hypertrophy. Ultrasound did not reveal and deep vein thrombosis and as patient was not keen for further surgical intervention, she did not proceed with further venous insufficiency Ultrasound Duplex.

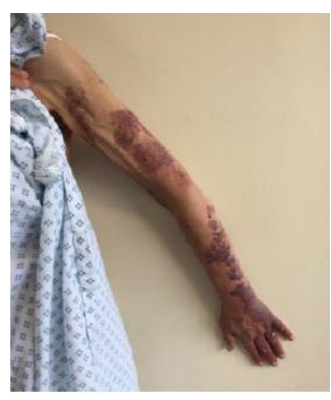

Figure1. Left upper limb portwine stains

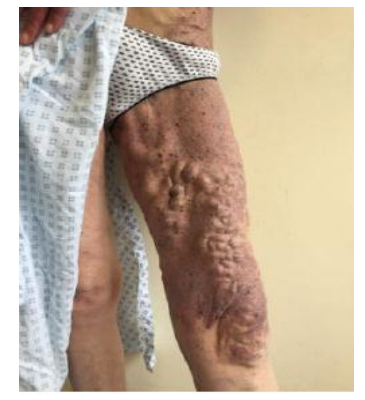

Figure2. Left lower limb port wine stains, hypertrophy and varicosities 


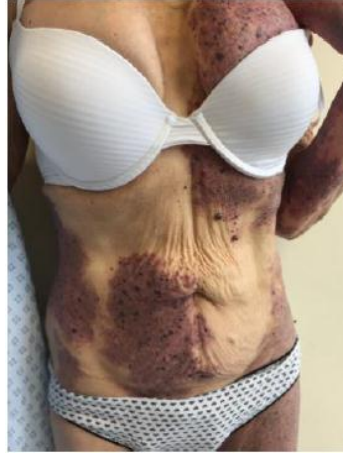

Figure3. Port wine stains over anterior torso

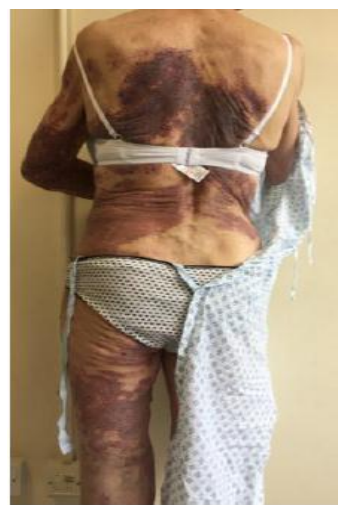

Figure4. Port wine stains over posterior torso

\section{DISCUSSION}

KTS affects men and women equally and is not limited to any racial group. Several molecular characteristics have been identified-including a translocation of chromosome 8q22.3 and 14q13 [2], which is associated with vascular and tissue overgrowth syndrome while others have found a terminal deletion of $2 \mathrm{q} 37.3$ in a patient with KTS [3]. Another study identified found an association of the vascular and/or overgrowth gene location on chromosome 18 [4].

KTS is typically characterized by a clinical triad of extremity varicosities, cutaneous vascular malformations, and hypertrophy of soft tissues and long bones. In a study of 252 patients with KTS (136 females and 116 males), capillary malformations in the form of port wine stains were found in $98 \%$ of patients (246 patients), $72 \%$ of the patients had varicosities (182 patients), and an incidence of soft tissue hypertrophy in $67 \%$ of the patients (170 patients). From the results, the student concluded that only 63\% (159 patients) of the patients studied had the classic clinical triad while a much fewer number of 93 patients (or $37 \%$ ) had two out of the three features of KTS [5].

Most KTS patients present with unilateral lower limb involvement (85\%) while $12.5 \%$ present with bilateral lower limb involvement and rarely, crossed bilateral involvement in $2.5 \%$ of the patients [6]. Hence, some has proposed the method of using a venous ultrasound duplex study as well as nuclear and radiological imaging to further confirm the diagnosis, as the clinical triad may not be present in all patients [7]. A venous ultrasound duplex study will not only help to confirm the presence and determine the extent of affected vessels, but also assess for complications of venous thrombosis. To differentiate between KTS and PWS, segmental limb pressure and ankle brachial index studies can be done to rule out the presence of arteriovenous shunts which is characteristic of PWS and not present in KTS [5]. With age, symptoms of varicosities and pain may worsen and can progress to the most severe complication of venous thromboembolism and pulmonary embolism [8]

Additional imaging to assess complications of KTS such as plain X-ray for imaging of long bones in patients with limb length discrepancy. Another useful tool for non-radiological or noncontrast imaging which would allow for better imaging of the different tissues would be a magnetic resonance (MR) scan or a MR angiography. It has the additional benefit of identifying lymphedema.

A multidisciplinary approach as well a nonsurgical approach has been the mainstay of KTS management. Treatment is largely symptomatic control and complication prevention. Complications such as venous thromboembolism and cellulitis through anticoagulation and the use of compression stockings [9]. Surgery is rarely indicated unless only in symptomatic patients who may require epiphyseodesis, ligation or stripping of incompetent veins. Additional surgical technique also includes subfascial endoscopic perforator surgery (SEPS).

\section{CONCLUSION}

Klippel Trenaunay Syndrome (KTS) is a rare, sporadic vascular malformation characterised by its clinical triad of: port wine stains, varicosities and limb hypertrophy. A multidisciplinary approach is the mainstay of KTS management and treatment is largely symptomatic control and complication prevention.

\section{REFERENCES}

[1] Pedro Ronaldo, LeyreAguado, Antonio Martinez-Cuesta, Diagnosis and management of extensive vascular malformations of the lower limb, American Academy of Dermatology. November 2011; 893-906 
[2] Q. Wang, A.A. Timur, P. Szafranski, A. Sadgephour, V. Jurecic, J. Cowell, A. Baldini, D.J. Driscoll, Identification and molecular characterization of de novo translocationt $(8 ; 14)(\mathrm{q} 22 ; \mathrm{q} 13)$ associated with a vascular and tissue overgrowth syndrome, Cytognet Cell Genetics. 2001; 95 (3-4); 183-188

[3] Hassan Vahidnezhad, Leila Youssefian, JouniUitto, Klippel Trenaunay Syndrome belongs to the PIK3CA-related overgrowth spectrum (PROS), Experimental Dermatology. 2015;25;17-19

[4] Anil, Timur, Azita Sadgephour, Michael Graf, Stuart Schwartz, Eric D. Libby Y, Davide J. Driscoll and Qing Wang, Identification and Molecular Characterization of a de novo Supernumerary Ring Chromosome 18 in a Patient with Klippel-Trenaunay Syndrome, Annals of Human Genetics.2004; 68; 353-261

[5] Jacob AG, Driscoll DJ, Shaughnessy WJ, Stanson AW, Clay RP, Gloviczki P,
KlippelTrenaunay syndrome: spectrum and management, Mayo Clinic Proceedings.1998;73(1);28-36

[6] Akira Baba, Shinji Yamazoe, Yum Okuyama, Kanichiro Shimizu, Yuko Kobashi, Yosuke Nozawa, Yohei Munetomo, Takuji Mogami, A rare presentation of Klippel-Trenaunay syndrome with bilateral lower limbs, Journal of Surgical Case Reports. 2017; 2; 1-3

[7] Rashid Rasheed, Durr-e-Sabih, Muhammad Kashif Rahim, Najam Uddin, Klippel Trenaunay Syndrome, Journal of the College of Physicians and Surgeons Pakistan. 2009 ;19 (11); 729-731

[8] Philippe A. Capraro, Jay Fisher, KlippelTrenaunay Syndrome, Plastic and Reconst ructive Surgery.2002; 109 (6); 2052-2060

[9] Deepak Sharma, SachinLamba, AakashPandita, SwetaShrasti, Klippel-Trenaunay Syndrome- A Very Rare and Interesting Syndrome, Clinical Medicine Insights, Circulatory, Respiratory and Pulmonary Medicine. 2015; 9; 1-3

Citation: Wei Zhi MAK, Qiantai HONG, Sadhana CHANDRASEKAR, Glenn Wei Leong TAN, Zhiwen Joseph LO. Klippel-Trenaunay Syndrome: A Case Report and Literature Review. ARC Journal of Surgery.2019; 5(2):1-3. DOI: http://dx.doi.org/10.20431/2455-572X.0502001

Copyright: (C) 2019 Authors. This is an open-access article distributed under the terms of the Creative Commons Attribution License, which permits unrestricted use, distribution, and reproduction in any medium, provided the original author and source are credited. 